Return to the Manage Active Submissions page at http://spie.org/app/submissions/tasks.aspx and approve or disapprove this submission. Your manuscript will not be published without this approval. Please contact author help@spie.org with any questions or concerns.

\title{
Numerical Modeling of Time-Resolved Photocurrent in Organic Semiconductor Films
}

\author{
Brian Johnson, Keshab Paudel, Mark J. Kendrick, and Oksana Ostroverkhova \\ Department of Physics, Oregon State University, Corvallis, OR 97331
}

\begin{abstract}
We performed numerical simulations of transient photocurrents in organic thin films, in conjunction with experiments. This enabled us to quantify the contribution of multiple charge generation pathways to charge carrier photogeneration, as well as extract parameters that characterize charge transport, in functionalized anthradithiophene (ADT-TES-F) films prepared using two different deposition methods: drop casting on an untreated substrate and spin casting on a pentafluorobenzenethiol (PFBT)-treated substrate. These deposition methods yielded polycrystalline films with considerably larger grain sizes in the case of the spin cast film. In both drop cast and spin cast films, simulations revealed two competing charge photogeneration pathways: fast charge generation on a picosecond (ps) or sub-ps time scale with efficiencies below $10 \%$, and slow charge generation, on the time scale of tens of nanoseconds, with efficiencies of $11-12 \%$ in drop cast and $50-60 \%$ in spin cast films, depending on the applied electric field. The total charge photogeneration efficiency in the spin cast sample was $59-67 \%$ compared to $14-20 \%$ in the drop cast sample, whereas the remaining $33-41 \%$ and $80-86 \%$, respectively, of the absorbed photon density did not contribute to charge carrier generation on these time scales. The spin cast film also exhibited higher hole mobilities, lower trap densities, shallower traps, and lower charge carrier recombination, as compared to the drop cast film. As a result, the spin cast film exhibited higher photocurrents despite a considerably lower film thickness (and thus reduced optical absorption and cross section of the current flow).
\end{abstract}

\section{INTRODUCTION}

Organic semiconductors are of interest as an alternative to inorganic semiconductors for many (opto)electronic applications due to their ease of fabrication, low cost, and tunable properties. A considerable research effort has been applied to characterizing charge photogeneration and transport, as well as structure-property relationships, in small-molecule and polymeric materials. ${ }^{1,2}$ One promising class of organic materials for a variety of (opto)electronic applications is solution-processable small-molecule organic semiconductors with high charge carrier mobilities and strong photoresponse. Examples of such materials are functionalized anthradithiophene (ADT) derivatives which display fast photoresponse, thin-film transistor (TFT) charge carrier (hole) mobilities as high as $\sim 1.5 \mathrm{~cm}^{2} /(\mathrm{Vs})$, high photoconductivity under continuous wave excitation, and a solid state packing controlled by the functionalization of the molecule and by the film deposition method..$^{3,4}$ This paper investigates variation in charge photogeneration and transport characteristics in thin films of fluorinated ADT functionalized with triethylsilylethynyl (TES) side groups (ADT-TES-F) under two different deposition methods that yield different film morphologies.

One of the difficulties in establishing physical mechanisms of photoexcited charge carrier dynamics in organic films is that experimentally measured photocurrents include contributions of a variety of processes that are challenging to disentangle, so that several different experimental techniques need to be applied to the same film in order to study each process separately. Numerical modeling of the photocurrent dynamics may provide a valuable insight into the relative contribution of each process to the photocurrent, thus avoiding multiple experiments. A considerable amount of such work has been done in polymer-based films, including Monte Carlo simulations of hopping conduction of photoexcited carriers, ${ }^{5-7}$ numerical modeling of the time-resolved photocurrent dynamics on various time scales, ${ }^{8-10}$ and modeling of the photocurrent in solar cells. ${ }^{5,11-15}$

In this paper, we present numerical modeling of nanosecond (ns) time-scale time-resolved photocurrent dynamics ${ }^{16}$ in ADT-TES-F films deposited by drop casting onto an untreated substrate and by spin casting on a pentafluorobenzenethiol (PFBT)-treated substrate. The choice of ADT-TES-F for numerical simulations was

8830 - 67 V. 1 (p.1 of 8) / Color: No / Format: Letter / Date: 7/29/2013 1:30:34 PM

SPIE USE: DB Check, Prod Check, Notes: 
Return to the Manage Active Submissions page at http://spie.org/app/submissions/tasks.aspx and approve or disapprove this submission. Your manuscript will not be published without this approval. Please contact author help@spie.org with any questions or concerns.

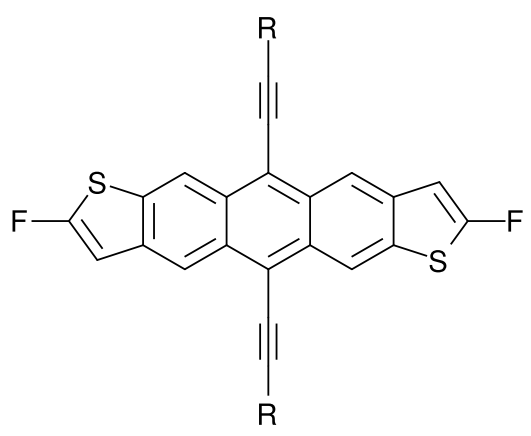

Figure 1. The molecular structure of ADT-R-F. In our study, R=TES.

motivated by our extensive previous work with this material, , ,16-18 as well as work of other groups on TFT characteristics and film structure and morphology of ADT-TES-F films depending on the film preparation method. ${ }^{3,19}$ This enabled us to check results of the numerical modeling against various experimentally observed trends in time-resolved photoluminescence (PL), photoconductivity, and charge carrier mobility. ${ }^{4,18,20,21}$ We quantified contributions of multiple pathways of charge photogeneration to ns time-scale photocurrent which include fast formation of spatially separated charge carriers and slow charge carrier photogeneration via Frenkel exciton dissociation. Additionally, we obtained parameters pertaining to subsequent transport of photoexcited charge carriers such as charge carrier mobilities, charge trapping, and recombination properties. ${ }^{16,20,21}$

\section{EXPERIMENT}

ADT-TES-F films were drop cast from toluene onto glass substrates patterned with interdigitated $\mathrm{Cr} / \mathrm{Au}$ electrodes with an $L=25 \mu \mathrm{m}$ gap between electrodes. The details of the drop cast sample preparation have been reported elsewhere. ${ }^{21}$ Additionally, ADT-TES-F films were spin cast at $3000 \mathrm{rpm}$ for 30 seconds from $30 \mathrm{mM}$ solutions in chlorobenzene onto similar substrates that had been treated with PFBT, which has been shown to improve film crystallinity and enhance hole mobility in ADT-TES-F TFTs. ${ }^{3,22}$ Both film preparation methods resulted in polycrystalline films, as confirmed by x-ray diffraction ${ }^{17,19,21}$ and optical imaging. The HOMO (LUMO) energies of ADT-TES-F measured using differential pulse voltammetry are 5.35 (3.05) eV. Voltage was applied to the samples and dark current was measured using a Keithley 237 source-measure unit. The average applied electric field (calculated as $E=V / L$ ) was kept between 20 and $80 \mathrm{kV} / \mathrm{cm}$. The transient photocurrents were measured using a $50 \mathrm{GHz}$ digital sampling oscilloscope (Tek80E01) with a broadband amplifier (Centellax UAOL65VM) after excitation with a $355 \mathrm{~nm}(3.49 \mathrm{eV}), 0.18 \mu \mathrm{J} / \mathrm{cm}^{2}$ (drop cast) or $0.4 \mu \mathrm{J} / \mathrm{cm}^{2}$ (spin cast), 500 ps pulsed laser beam (cavity Q-switched frequency-tripled Nd:YAG laser, $44.6 \mathrm{kHz}$, from Nanolase, Inc.). The time resolution of the system was $\sim 0.6 \mathrm{~ns}$, limited by the laser pulse width and jitter. For appropriate comparison to simulation, the current values were converted to corresponding current densities, assuming an active layer depth of $d=1 \mu \mathrm{m}$ for the drop cast films and $d=200 \mathrm{~nm}$ for the spin cast films, based on the average thickness of our films.

\section{THEORY}

A detailed overview of the model used, which describes transient photocurrent dynamics in both pristine materials and their donor-acceptor composites, can be found elsewhere. ${ }^{16}$ Briefly, due to the large size $(L=25 \mu \mathrm{m})$ of our devices, we have assumed that the electric field and current densities can be averaged over the device. This reduces the common drift-diffusion model ${ }^{11}$ to a drift-only current model. We used an effective medium approach for the mobilities with $\mu_{n}$ and $\mu_{p}$ representing the mobility of electrons and holes, respectively. We modeled both electron and hole trapping with trapping rates $B_{n}$ and $B_{p}$ into trap densities of $N_{n}$ and $N_{p}$, respectively. Since holes are the majority carriers in our devices and are more likely to encounter shallow traps, we modeled hole detrapping with a Millers-Abraham's approach defined by an average trap depth $\Delta$.

\section{0 - 67 V. 1 (p.2 of 8) / Color: No / Format: Letter / Date: 7/29/2013 1:30:34 PM}


Return to the Manage Active Submissions page at http://spie.org/app/submissions/tasks.aspx and approve or disapprove this submission. Your manuscript will not be published without this approval. Please contact author_help@spie.org with any questions or concerns.

We previously established that even in pristine materials charge carrier photogeneration followed multiple pathways. ${ }^{16}$ The first, denoted here as "Spatially Separated Carriers" (SSC) pathway, describes charge carriers created on ps or sub-ps time-scales, ${ }^{23}$ most likely via hot exciton dissociation. The second pathway is a Frenkel exciton (FE) that can dissociate to free carriers with a rate $k_{d i s s, F E}$. We have modeled the FE dissociation using the Onsager-Braun formalism, defined by an initial pair separation $a_{F E}$ and a recombination rate $k_{r, F E}$. As shown below, the major part of photoexcitation in drop cast films, and a large part of it in spin cast films, does not follow either of these two pathways. Instead, it decays to the ground state through processes that do not produce charge carriers on our time scales, such as PL emission.

Given these considerations, we obtained the following equations:

$$
\begin{gathered}
\frac{d n_{f}}{d t}=\xi_{S S C}(E) G(t)+k_{d i s s, F E} X_{F E}-\gamma n_{f} p_{f} \\
-B_{n}\left(N_{n}-n_{t}\right) n_{f}-B_{n_{f} p_{t}} n_{f} p_{t} \\
\frac{d n_{t}}{d t}=B_{n}\left(N_{n}-n_{t}\right) n_{f}-B_{p_{f} n_{t}} n_{t} p_{f} \\
\frac{d p_{f}}{d t}=\xi_{S S C}(E) G(t)+k_{d i s s, F E} X_{F E}+B_{p_{t}} p_{t}-\gamma n_{f} p_{f} \\
-B_{p}\left(N_{p}-p_{t}\right) p_{f}-B_{p_{f} n_{t}} n_{t} p_{f} \\
\frac{d p_{t}}{d t}=B_{p}\left(N_{p}-p_{t}\right) p_{f}-B_{n_{f} p_{t}} n_{f} p_{t}-B_{p_{t}} p_{t} \\
\frac{d X_{F E}}{d t}=\xi_{F E}(E) G(t)-k_{d i s s, F E} X_{F E} \\
-k_{r, F E} X_{F E} \\
k_{d i s s, F E}(E, T)=\frac{3 \gamma J_{1}(2 \sqrt{-2 b})}{4 \pi a_{F E}^{3} \sqrt{-2 b}} \exp \left(-\frac{E_{B, F E}}{k_{B} T}\right) \\
b=\left(e^{3}|E|\right) /\left(8 \pi \epsilon_{0} \epsilon_{r}\left(k_{B} T\right)^{2}\right) \\
J=e E\left(\mu_{n}(E) n_{f}+\mu_{p}(E) p_{f}\right) \\
\mu_{n(p)}(E)=\mu_{n 0(p 0)} \exp \left(\gamma_{n(p)} \sqrt{E}\right)
\end{gathered}
$$

where $t$ is the time, $e$ is the fundamental charge, $\epsilon_{0}$ is the permittivity of free space, $\epsilon_{r}$ is the relative permittivity of the film (taken to be 3). Variables $p(n)_{f}$ correspond to free hole (electron) density, $p(n)_{t}$ are the trapped hole (electron) density, and $X_{F E}$ is the exciton density for the FE. $J$ is the total current density, $E$ is the applied electric field, $G$ is the photoexcitation rate described below (Eq. 11), and $\xi_{S S C(F E)}$ are fractions of the photoexcitation that result in charge photogeneration via the SSC pathway and in FE formation. $k_{d i s s, F E}$ is the dissociation rate for the $\mathrm{FE}$, and $k_{r, F E}$ is the recombination rate for the FE. $E_{B, F E}$ is the binding energy of the $\mathrm{FE}$ given by $E_{B, F E}=e^{2} /\left(4 \pi \epsilon_{0} \epsilon_{r} a_{F E}\right)$, where $a_{F E}$ is the initial separation between charge carriers for the FE. Equations 1 and 3 are the coupled drift equations for electron and hole densities, with multiple charge generation paths as described above and bimolecular recombination of free carriers to the ground state with the Langevin rate constant of Eq. 6. Bimolecular recombination with a formation of the FE was also considered, ${ }^{24}$ but produced a negligible effect on the photocurrent dynamics and was omitted. Equations 2 and 4 describe the trapping, trap assisted recombination, and hole detrapping of carriers, governed by their respective rates. Equation 5 describes the dynamics of the dissociation and recombination of $\mathrm{FE}$. The dissociation rate of the $\mathrm{FE}$ is given by Eq. 7 and is assumed to follow the Onsager-Braun formalism. ${ }^{25} \mu_{p 0}\left(\mu_{n 0}\right)$ and $\gamma_{p}\left(\gamma_{n}\right)$ are Poole-Frenkel model parameters characterizing zero-field mobility and electric field dependence of mobility for holes (electrons).

The excitation is modelled as a perfect 500 ps Gaussian pulse that is uniform in space over our device with a total area of $\int_{-\infty}^{\infty} G(t)=N_{p h}$, where $N_{p h}$ is the total density of absorbed photons. (Under our experimental conditions, the two-dimensional density of the absorbed photons is $2.9 \times 10^{11} \mathrm{~cm}^{-2}$ and $1.9 \times 10^{11} \mathrm{~cm}^{-2}$ for drop cast and spin cast films, respectively.) This gives

$$
G(t)=N_{p h} \frac{2 \sqrt{\ln 2}}{\tau \sqrt{\pi}} * \exp \left(-4 \ln 2 \frac{\left(t-t_{F W T M} / 2\right)^{2}}{\tau^{2}}\right)
$$

\section{0 - 67 V. 1 (p.3 of 8) / Color: No / Format: Letter / Date: 7/29/2013 1:30:34 PM}


Return to the Manage Active Submissions page at http://spie.org/app/submissions/tasks.aspx and approve or disapprove this submission. Your manuscript will not be published without this approval. Please contact author_help@spie.org with any questions or concerns.

\begin{tabular}{l|c|c} 
Parameter $(\mathrm{u})$ & Drop cast ADT-TES-F & Spin cast ADT-TES-F \\
\hline$\mu_{n, 0}\left(\mathrm{~cm}^{2}(\mathrm{Vs})^{-1}\right)$ & 0.093 & 0.12 \\
$\mu_{p, 0}\left(\mathrm{~cm}^{2}(\mathrm{Vs})^{-1}\right)$ & 0.60 & 1.2 \\
$\gamma_{n}(\mathrm{~cm} / \mathrm{V})^{1 / 2}$ & $1.8 \times 10^{-3}$ & $2.8 \times 10^{-4}$ \\
$\gamma_{p}(\mathrm{~cm} / V)^{1 / 2}$ & $3.1 \times 10^{-4}$ & $5.4 \times 10^{-6}$ \\
$\Delta(\mathrm{meV})$ & 29 & 24 \\
$\left.N_{n}(\mathrm{~cm})^{-3}\right)$ & $7.1 \times 10^{18}$ & $4.8 \times 10^{18}$ \\
$N_{p}\left(\mathrm{~cm}^{-3}\right)$ & $2.8 \times 10^{18}$ & $4.9 \times 10^{17}$ \\
$B_{n} N_{n}\left(\mathrm{~s}^{-1}\right)$ & $5.1 \times 10^{11}$ & $7.5 \times 10^{11}$ \\
$B_{p} N_{p}\left(\mathrm{~s}^{-1}\right)$ & $3.9 \times 10^{10}$ & $6.5 \times 10^{10}$ \\
$B_{n_{f}} p_{t}\left(\mathrm{~cm}^{3} \mathrm{~s}^{-1}\right)$ & $1.4 \times 10^{-3}$ & $1.8 \times 10^{-4}$ \\
$B_{p_{f}} n_{t}\left(\mathrm{~cm}^{3} \mathrm{~s}^{-1}\right)$ & $2.1 \times 10^{-5}$ & $3.1 \times 10^{-7}$ \\
$a_{F E}\left(\mathrm{~nm}^{-1}\right)$ & 1.18 & 1.06 \\
\hline
\end{tabular}

Table 1. Parameter values extracted from experimental data for drop cast and spin cast ADT-TES-F films using a simulation with a system of Eqs.(1-11). Parameter descriptions are given in the text.

where $t_{F W T M}$ is the full width at a tenth of maximum of the Gaussian pulse and so $G(t=0)=0.1 G_{\max }$ where $G_{\max }$ is the photon density at the laser pulse maximum.

In order to obtain physically meaningful simulation results, realistic initial carrier densities are necessary. ${ }^{16}$ We considered as our boundary conditions thermionic injection with image charge effects at the electrode-organic interface. ${ }^{26,27}$ The injection barriers $\phi_{B}^{p}$ and $\phi_{B}^{n}$ for holes and electrons, respectively, were taken to be $0.25 \mathrm{eV}$ and $2.05 \mathrm{eV}$. We then assumed that the carrier densities had reached a steady state $(d p(n) / d t=0)$ before the laser pulse excitation. This allowed us to solve ${ }^{27}$ for the average densities at $t=0:^{16}$

$$
\begin{aligned}
n_{f}^{0} & =\frac{J_{\text {Dark }}(E)}{e E} \frac{e^{-\frac{\phi_{B}^{n}}{k_{B} T}}}{\mu_{n} e^{-\frac{\phi_{B}^{n}}{k_{B} T}}+\mu_{p} e^{-\frac{\phi_{B}^{p}}{k_{B} T}}} \\
p_{f}^{0} & =\frac{J_{\text {Dark }}(E)}{e E} \frac{e^{-\frac{\phi_{B}^{p}}{k_{B} T}}}{\mu_{n} e^{-\frac{\phi_{B}^{n}}{k_{B} T}}+\mu_{p} e^{-\frac{\phi_{B}^{p}}{k_{B} T}}} \\
n_{t}^{0} & =\left(B_{n} N_{n} n_{f}^{0}\right) /\left(B_{n} n_{f}^{0}+B_{p_{f}} n_{t} p_{f}^{0}\right) \\
p_{t}^{0} & =\left(B_{p} N_{p} p_{f}^{0}\right) /\left(B_{p} p_{f}^{0}+B_{n_{f} p_{t}} n_{f}^{0}+B_{p_{t}}\right)
\end{aligned}
$$

where $J_{\text {Dark }}(E)$ is the measured dark current density at the applied electric field $E$.

The system of Eqs. 1-11 with initial conditions Eqs. 12-15 was solved numerically in MATLAB using the built in ode15s function. The simulated transients were then fit to the data using the non-linear optimization package NLOPT. ${ }^{28}$ The fitting was accomplished through minimizing one of two objective functions:

$$
\begin{aligned}
& f_{1}(\vec{x})=\sum_{n}\left(J_{\text {Data }}\left(t_{n}\right)-J_{\text {Sim }}\left(t_{n}, \vec{x}\right)\right)^{2} \\
& f_{2}(\vec{x})=\sum_{m}\left(1-r_{m}^{2}\right)
\end{aligned}
$$

where $\vec{x}$ is the vector input to the simulation function that incorporates all varied parameters of the model, $t_{n}$ is the nth discrete time value, $J_{\text {Data }}\left(t_{n}\right)$ is the experimentally measured total current at $t_{n}, J_{\text {Sim }}\left(t_{n}, \vec{x}\right)$ is the simulated total current at $t_{n}$, and $r_{m}^{2}$ is the coefficient of determination of the simulated fit for the mth applied electric field. Using these objective functions, we obtained sets of parameters that describe experimentally measured photocurrent transients at all applied electric fields in the studied range.

\section{0 - 67 V. 1 (p.4 of 8) / Color: No / Format: Letter / Date: 7/29/2013 1:30:34 PM}


Return to the Manage Active Submissions page at http://spie.org/app/submissions/tasks.aspx and approve or disapprove this submission. Your manuscript will not be published without this approval. Please contact author_help@spie.org with any questions or concerns.
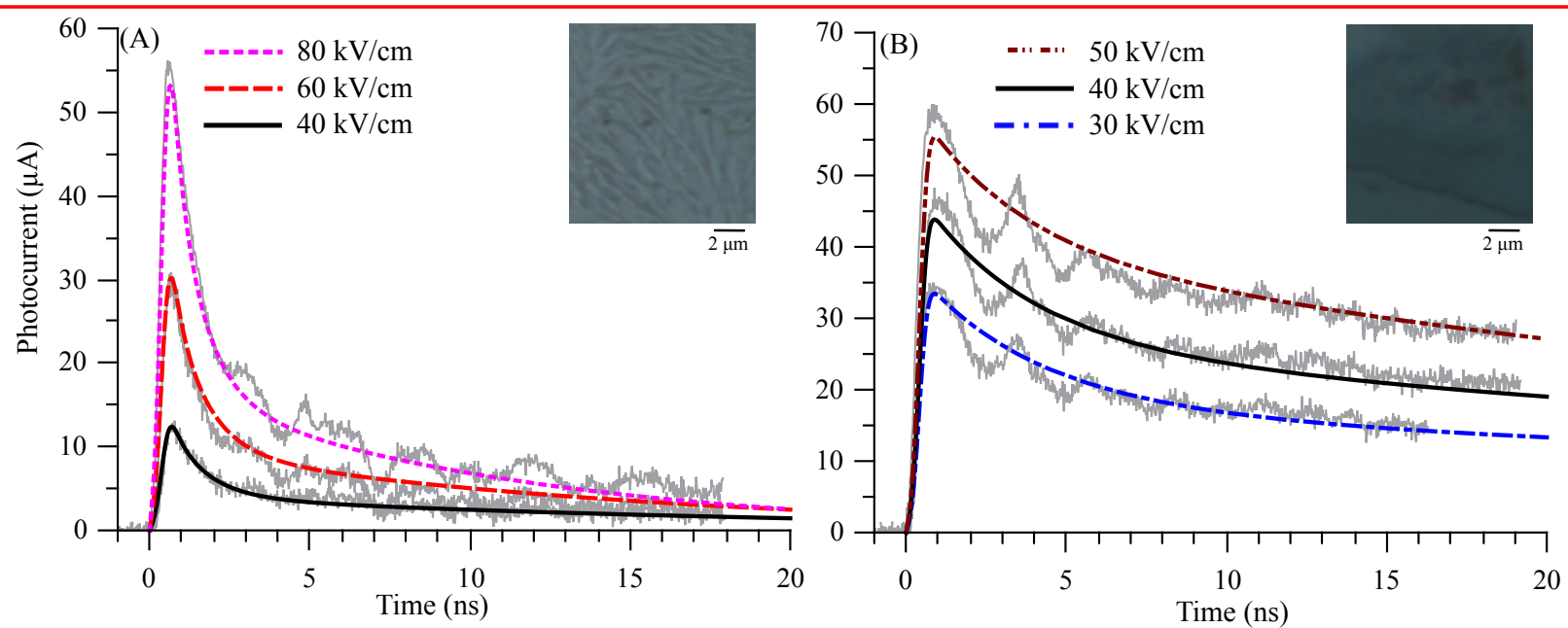

Figure 2. Experimental and simulated photocurrents from (A) drop cast and (B) spin cast ADT-TES-F films at varied electric fields. Insets show optical images of drop cast and spin cast films used in our experiments at 50x magnification.
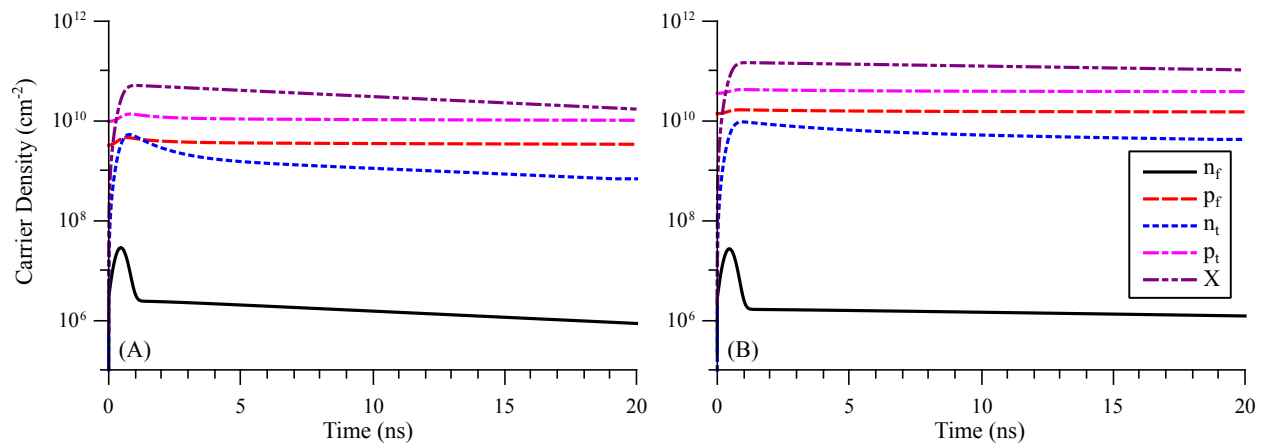

Figure 3. Simulated time evolution of densities of Frenkel exciton $(X)$, free $\left(p_{f}\right)$ and trapped $\left(p_{t}\right)$ holes, and free $\left(n_{f}\right)$ and trapped $\left(n_{t}\right)$ electrons for a drop cast film (A) and a spin cast film (B) at $40 \mathrm{kV} / \mathrm{cm}$.

\section{RESULTS AND DISCUSSION}

The process of arriving at fits for drop cast pristine ADT-TES-F films has been described elsewhere. ${ }^{16}$ The same process has been used to fit data from an ADT-TES-F film spin cast on PFBT-treated substrates, the results of which we now present.

The simulated photocurrents obtained for the drop and spin cast samples using the parameter values reported in Table 1 are presented in Figure 2. Time evolution of exciton and charge carrier densities in these samples that resulted in the photocurrent transients in Fig. 2 at an applied electric field of $40 \mathrm{kV} / \mathrm{cm}$ is shown in Fig. 3. The spin cast film exhibited higher photocurrent values as compared to those in drop cast films at a given applied electric field despite a considerably lower film thickness (and thus, reduced optical absorption and crosssection of the current flow). We thus expect to see significantly higher charge photogeneration efficiency and hole mobility in the spin cast films, a result supported by previous TFT work on ADT-TES-F films spin cast onto PFBT-treated substrates with Au electrodes. ${ }^{3,22}$ Indeed, our simulations revealed that higher photocurrents in thin spin cast films can be partially attributed to an increase in hole mobility ( $\mu_{p 0}$ in Table 1$)$ and to a significant increase in the charge photogeneration efficiency (Fig. 4), especially that of the FE pathway (Fig. 4(a)). The larger zero-field hole mobility value $\left(\mu_{p 0}\right.$ in Table 1$)$, smaller Poole-Frenkel mobility electric field dependence factor $\left(\gamma_{p}\right.$ in Table 1$)$, lower trap density $\left(N_{p}\right.$ in Table 1$)$, and lower average trap depth $(\Delta$ in Table 1$)$ for the spin cast film are consistent with lower disorder due to larger crystallite sizes in this film, as compared to those in the drop cast sample, as seen from the optical images in the insets of Fig. 2.

A comparison of the charge photogeneration efficiencies via $\operatorname{SSC}\left(\xi_{S S C}\right)$ and $\mathrm{FE}$ dissociation pathways $\left(\xi_{F E} *\right.$ $\eta_{F E}$, where $\eta_{F E}=k_{d i s s, F E} /\left(k_{d i s s, F E}+k_{r, F E}\right)$ is the efficiency of FE dissociation) is shown in Fig. 4. Both $\xi_{S S C}$ 
Return to the Manage Active Submissions page at http://spie.org/app/submissions/tasks.aspx and approve or disapprove this submission. Your manuscript will not be published without this approval. Please contact author help@spie.org with any questions or concerns.
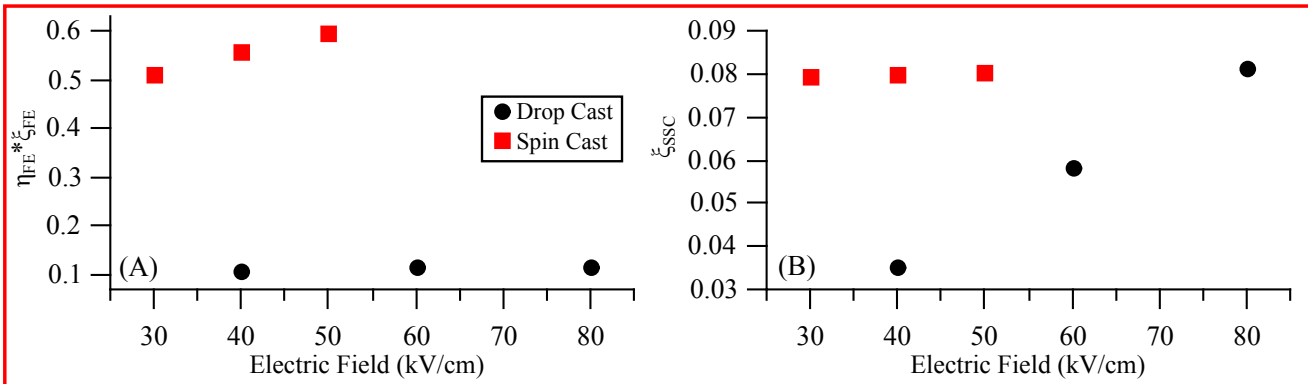

Figure 4. A comparison of the (A) FE charge generation pathway efficiency and (B) SSC charge generation pathway efficiency in spin cast and drop cast ADT-TES-F films.

and $\xi_{F E} * \eta_{F E}$ were higher in the spin cast film; however, the most pronounced difference between the drop cast and spin cast films was in the efficiency of the $\mathrm{FE}$ formation $\xi_{F E}$, which was significantly larger in the spin cast film ( e.g. $79 \%$ in the spin cast film, as compared to $20 \%$ in the drop cast film, at $40 \mathrm{kV} / \mathrm{cm}$, Fig. 5). Additionally, the simulations revealed that the FE dynamics differed significantly between the drop cast and spin cast films (X in Fig. 3), as the lifetime of the FE in the spin cast film (46-67ns, depending on the electric field) was 3-4 times longer than that in the drop cast film. ${ }^{16}$ This suggests that recombination of the charge-generating $\mathrm{FE}$ in ADT-TES-F films occurs largely at the grain boundaries; it is less efficient in our spin cast film due to its larger crystallite sizes as compared to those in a drop cast film. This is in contrast to the PL-emitting ADT-TES-F exciton, which exhibits a considerably shorter PL lifetime in spin cast films ${ }^{29}$ as compared to drop cast films, ${ }^{4,21}$ and properties of which are related to exciton delocalization within the crystallite. ${ }^{17}$

The total amount of charge generated at ns time scales, given by $\xi_{S S C}+\xi_{F E} * \eta_{F E}$, was higher by a factor of $\sim 3-4$, depending on the electric field, in the spin cast film. As discussed above, this is mostly due to the differences in the $\mathrm{FE}$ formation efficiency, whereas the efficiency of $\mathrm{FE}$ dissociation, $\eta_{F E}$, was comparable in the spin cast and drop cast samples. A schematic representation of the distribution of photoexcitation among various relaxation pathways is shown in Figure 5.

The initial decay of the photocurrent (Fig. 2) in the spin cast sample was much slower than that in the drop cast sample. Our simulation results indicate that this difference is primarily due to the different charge trapping and recombination characteristics of the two films (Fig. 3). The free hole-trapped electron trap-assisted recombination rate $B_{p_{f} n_{t}}$ is particularly important. ${ }^{16}$ The considerably lower value of $B_{p_{f} n_{t}}$ in the spin cast film indicates that holes are less likely to encounter trapped electrons, and less likely to recombine if they do encounter each other, as compared to that in the drop cast film. This suggests that a large number of trap states in ADT-TES-F films form on grain boundaries, which are considerably reduced by the PFBT treatment of the substrate with Au electrodes combined with the spin cast deposition method. ${ }^{22}$ This manifests in the reduced trap densities $N_{p(n)}$ and the trap-assisted recombination rates $B_{p_{f} n_{t}}\left(B_{n_{f} p_{t}}\right)$, as well as shallower traps $(\Delta$ in Table 1) in the spin cast film as compared to the drop cast film. This process competes with the higher hole mobility causing carriers to encounter traps more often, as evidenced by the values of bulk trapping rates $B_{n} N_{n}$ and $B_{p} N_{p}$ shown in Table 1 . The overall effect is an increased speed of initial charge trapping and slower relaxation of the free and trapped charge densities in spin cast films (Fig.3).

\section{CONCLUSIONS}

We applied a model ${ }^{16}$ that allows us to quantify the contribution of multiple charge generation pathways to charge carrier photogeneration, as well as extract parameters that characterize charge transport, to organic semiconductor films prepared using two different deposition methods: drop casting on an untreated substrate and spin casting on a PFBT-treated substrate. In both films, simulations revealed two competing charge photogeneration pathways: fast charge generation on a ps or sub-ps time scale with efficiencies below $10 \%$, and slow charge generation, on the time scale of tens of nanoseconds, with efficiencies of $11-12 \%$ in drop cast and of $50-60 \%$ in spin cast films, depending on the applied electric field. The total charge photogeneration efficiency in the spin cast sample was $59-67 \%$ compared to $14-20 \%$ in the drop cast sample, whereas the remaining $33-41 \%$ and $80-86 \%$, respectively, of the photoexcitation did not contribute to charge carrier generation on these time

\section{0 - 67 V. 1 (p.6 of 8) / Color: No / Format: Letter / Date: 7/29/2013 1:30:34 PM}


Return to the Manage Active Submissions page at http://spie.org/app/submissions/tasks.aspx and approve or disapprove this submission. Your manuscript will not be published without this approval. Please contact author_help@spie.org with any questions or concerns.

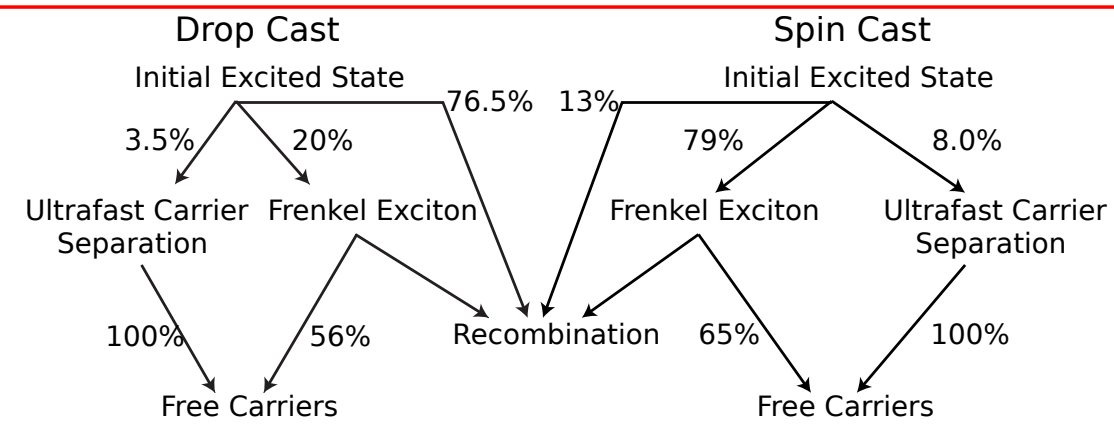

Figure 5. Flowcharts showing distribution of photoexcitation among various relaxation pathways in drop cast and spin cast films. The values of each pathway efficiency are exemplars from the $40 \mathrm{kV} / \mathrm{cm}$ fits multiplied by $100 \%$.

scales. The spin cast sample also exhibited higher hole mobilities, lower trap densities, shallower traps, and lower charge carrier recombination, as compared to those in the drop cast sample. Because of these properties, the spin cast film exhibited higher photocurrents than the drop cast film, despite its considerably lower thickness leading to a lower absorption and cross-section of the current flow.

\section{Acknowledgements}

We thank Prof. J.E. Anthony for ADT-TES-F and J. Ward and Prof. O. D. Jurchescu for helpful tips on the PFBT treatment of the substrates. This work was supported by the NSF grants DMR-0748671 (via CAREER program) and DMR-1207309.

\section{REFERENCES}

1. T. Clarke and J. Durrant, "Charge photogeneration in organic solar cells," Chem. Rev. 110(11), 6736, 2010.

2. B. Walker, C. Kim, and T.-Q. Nguyen, "Small molecule solution-processed bulk heterojunction solar cells," Chem. Mat. 23(3), 470, 2011.

3. D. J. Gundlach, J. E. Royer, S. K. Park, S. Subramanian, O. D. Jurchescu, B. H. Hamadani, A. J. Moad, R. J. Kline, L. C. Teague, O. Kirillov, C. A. Richter, J. G. Kushmerick, L. J. Richter, S. R. Parkin, T. N. Jackson, and J. E. Anthony, "Contact-induced crystallinity for high-performance soluble acene-based transistors and circuits," Nature Mater. 7(3), 216, 2008.

4. A. D. Platt, J. Day, S. Subramanian, J. E. Anthony, and O. Ostroverkhova, "Optical, fluorescent, and (photo)conductive properties of high-performance functionalized pentacene and anthradithiophene derivatives," J. Phys. Chem. C 113(31), 14006, 2009.

5. H. van Eersel, R. A. J. Janssen, and M. Kemerink, "Mechanism for efficient photoinduced charge separation at disordered organic heterointerfaces," Adv. Func. Mater. 22(13), 2700, 2012.

6. M. C. Heiber and A. Dhinojwala, "Dynamic monte carlo modeling of exciton dissociation in organic donoracceptor solar cells.," J. Chem. Phys. 137(1), 014903, 2012.

7. J. Nelson, "Diffusion-limited recombination in polymer-fullerene blends and its influence on photocurrent collection," Phys. Rev. B 67(15), 155209, 2003.

8. C. Soci, D. Moses, Q.-H. Xu, and A. Heeger, "Charge-carrier relaxation dynamics in highly ordered poly(pphenylene vinylene): Effects of carrier bimolecular recombination and trapping," Phys. Rev. B 72(24), 245204, 2005.

9. L. Kulikovsky, D. Neher, E. Mecher, K. Meerholz, H.-H. Hörhold, and O. Ostroverkhova, "Photocurrent dynamics in a poly(phenylene vinylene)-based photorefractive composite," Phys. Rev. B 69(12), 125216, 2004.

10. O. Ostroverkhova and K. D. Singer, "Space-charge dynamics in photorefractive polymers," J. Appl. Phys. 92(4), 1727, 2002.

11. I. Hwang, C. R. McNeill, and N. C. Greenham, "Drift-diffusion modeling of photocurrent transients in bulk heterojunction solar cells," J. Appl. Phys. 106(9), 094506, 2009. 
Return to the Manage Active Submissions page at http://spie.org/app/submissions/tasks.aspx and approve or disapprove this submission. Your manuscript will not be published without this approval. Please contact author_help@spie.org with any questions or concerns.

12. J.-T. Shieh, C.-H. Liu, H.-F. Meng, S.-R. Tseng, Y.-C. Chao, and S.-F. Horng, "The effect of carrier mobility in organic solar cells," J. Appl. Phys. 107(8), 084503, 2010.

13. L. Koster, E. Smits, V. Mihailetchi, and P. Blom, "Device model for the operation of polymer/fullerene bulk heterojunction solar cells," Phys. Rev. B 72(8), 085205, 2005.

14. T. Kirchartz, B. E. Pieters, K. Taretto, and U. Rau, "Mobility dependent efficiencies of organic bulk heterojunction solar cells: Surface recombination and charge transfer state distribution," Phys. Rev. B 80(3), 035334, 2009.

15. A. Petersen, A. Ojala, T. Kirchartz, T. Wagner, F. Wrthner, and U. Rau, "Field-dependent exciton dissociation in organic heterojunction solar cells," Phys. Rev. B 85(24), 245208, 2012.

16. B. Johnson, M. Kendrick, and O. Ostroverkhova, "Charge carrier dynamics in organic semiconductors and their donor-acceptor composites: numerical modeling of time-resolved photocurrent," submitted to J. Appl. Phys., 2013.

17. A. Platt, M. Kendrick, M. Loth, J. Anthony, and O. Ostroverkhova, "Temperature dependence of exciton and charge carrier dynamics in organic thin films," Phys. Rev. B 84(23), 235209, 2011.

18. W. E. B. Shepherd, A. D. Platt, D. Hofer, O. Ostroverkhova, M. Loth, and J. E. Anthony, "Aggregate formation and its effect on (opto)electronic properties of guest-host organic semiconductors," Appl. Phys. Lett. 97(16), 163303, 2010.

19. R. J. Kline, S. D. Hudson, X. Zhang, D. J. Gundlach, A. J. Moad, O. D. Jurchescu, T. N. Jackson, S. Subramanian, J. E. Anthony, M. F. Toney, and L. J. Richter, "Controlling the microstructure of solutionprocessable small molecules in thin-film transistors through substrate chemistry," Chem. Mat. 23(5), 1194, 2011.

20. W. E. B. Shepherd, A. D. Platt, M. J. Kendrick, M. A. Loth, J. E. Anthony, and O. Ostroverkhova, "Energy transfer and exciplex formation and their impact on exciton and charge carrier dynamics in organic films," J. Phys. Chem. Lett 2(5), 362, 2011.

21. M. J. Kendrick, A. Neunzert, M. M. Payne, B. Purushothaman, B. D. Rose, J. E. Anthony, M. M. Haley, and O. Ostroverkhova, "Formation of the donor-acceptor charge-transfer exciton and its contribution to charge photogeneration and recombination in small-molecule bulk heterojunctions," J. Phys. Chem. C 116(34), $18108,2012$.

22. J. W. Ward, M. a. Loth, R. J. Kline, M. Coll, C. Ocal, J. E. Anthony, and O. D. Jurchescu, "Tailored interfaces for self-patterning organic thin-film transistors," J. Mat. Chem. 22(36), 19047, 2012.

23. J. Day, S. Subramanian, J. E. Anthony, Z. Lu, R. J. Twieg, and O. Ostroverkhova, "Photoconductivity in organic thin films: From picoseconds to seconds after excitation," J. Appl. Phys. 103(12), 123715, 2008.

24. W. Tress, K. Leo, and M. Riede, "Optimum mobility, contact properties, and open-circuit voltage of organic solar cells: A drift-diffusion simulation study," Phys. Rev. B 85(15), 155201, 2012.

25. C. Braun, "Electric field assisted dissociation of charge transfer states as a mechanism of photocarrier production," J. Chem. Phys. 80, 4157, 1984.

26. J. Scott and G. Malliaras, "Charge injection and recombination at the metal-organic interface," Chem. Phys. Lett. 299, 115, 1999.

27. S. Lacic and O. Inganas, "Modeling electrical transport in blend heterojunction organic solar cells," J. Appl. Phys. 97(12), 124901, 2005.

28. S. G. Johnson. The NLOPT non-linear optimization package, version 2.3, 2012, see www.ab-initio.mit. edu/nlopt.

29. K. Paudel, B. Johnson, A. Neunzera, M. Thieme, J. Anthony, and O. Ostroverkhova in preparation for submission to J. Phys. Chem. C. , 2013. 